\title{
Filigrane
}

Écoutes psychothérapiques

\section{Présentation. L’avenir du clinicien II}

\section{Hélène Richard}

Volume 17, numéro 2, automne 2008

L’avenir du clinicien II

URI : https://id.erudit.org/iderudit/019414ar

DOI : https://doi.org/10.7202/019414ar

Aller au sommaire du numéro

Éditeur(s)

Revue Santé mentale au Québec

ISSN

1192-1412 (imprimé)

1911-4656 (numérique)

Découvrir la revue

Citer ce document

Richard, H. (2008). Présentation. L’avenir du clinicien II. Filigrane, 17(2), 3-5. https://doi.org/10.7202/019414ar d'utilisation que vous pouvez consulter en ligne.

https://apropos.erudit.org/fr/usagers/politique-dutilisation/ 


\title{
Présentation \\ L'avenir du clinicien II
}

\author{
hélène richard
}

$\mathrm{L}$ e premier dossier 2008 de Filigrane, «L'avenir du clinicien psychanalytique I», décrivait l'avenir du clinicien confronté aux contrecoups de l'idéalisation dont a été l'objet la psychanalyse au Québec durant les décennies 1960 et 1970. Seul son prestige social s'est terni, toutefois, la psychanalyse, elle, est demeurée bien vivante. Ce dossier en témoigne.

Dans le second dossier 2008, Filigrane examine l'avenir du clinicien confronté aux avatars du regain de popularité dont jouit, en ce moment dans plusieurs domaines professionnels, la pensée scientifique d'orientation positiviste. En quoi cette recrudescence de la résistance à la psychanalyse, venant tant du dehors que du dedans, influence-t-elle notre travail ? Quels sont alors les nouveaux visages de la psychanalyse clinique? les récentes modalités d'écoute de l'inconscient qu'a développées récemment le clinicien? Y a-t-il lieu d'avancer que «la psychothérapie pratiquée par les psychanalystes n'appauvrit pas la psychanalyse, [qu']elle étend les limites de son champ, défini par son cadre $^{1} »$ ?

Peut-on penser que le clinicien psychanalytique pourrait aujourd'hui jouer un nouveau rôle dans la cité en se mettant à l'écoute de la détresse de l'intervenant submergé, on le sait, par la dureté de son métier dans le secteur public des soins de santé et où sévit le regain de l'orientation positiviste, fût-il médecin «de première ligne », comme le dit la Réforme en santé mentale du Québec ? Comment, par ailleurs, rester à l'écoute d'une demande en composant avec les structures et règles du jeu en vigueur dans ce secteur public?

Ces questions, Filigrane a demandé à ses auteurs de les développer à même le quotidien de leur pratique clinique, car c'est de cette position que s'interroge son lectorat, qu'il est à l'affût de ressources de lucidité et d'inspiration. Les auteurs ont répondu, à leur manière, de la façon suivante.

Claudette Lafond invite le lecteur à réfléchir sur les manifestations de la résistance et du dogmatisme, à la fois chez les cliniciens et dans les politiques de santé mentale. Son propos met en évidence les répercussions de ces manifestations qui influencent le clinicien d'orientation psychodynamique. Il souligne l'hétérogénéité entre l'approche analytique et celles qui se veulent scientifiques d'inspiration positiviste, tentant ainsi de montrer l'incompatibilité des finalités poursuivies. À partir de ces constats, l'avenir du clinicien d'orientation analytique se concevrait par l'affirmation de son identité liée à l'héritage subversif de la psychanalyse et par l'obligation de rendre compte de son travail dans le social. 
Bernard Chervet, pour sa part, souligne le fait que la psychanalyse s'est saisie de l'utilisation très ancienne de la régression à des fins thérapeutiques. La tentative d'amélioration des protocoles de soins ne peut faire l'impasse sur la régression en tant qu'outil. Du point de vue des visées thérapeutiques, une valeur particulière est à reconnaître à la régression d'incidence. D'autres modalités de régressions sont aussi observables, de jour comme de nuit. Du fait de leur proximité avec celle spécifique des protocoles thérapeutiques, elles tendent à devenir des résistances à la guérison.

Pierre Lamoureux, quant à lui, rejoint certaines considérations qu'élabore Claudette Lafond. Le contexte social actuel, selon lui, conteste au clinicien les étayages professionnels qui définissent la pertinence de son apport. L'effraction d'une culture qui surinvestit la dimension du public dans le domaine privé de la cure subvertit cet espace aux fins de l'assimiler. Il s'agirait là d'une forme renouvelée de la résistance à la psychanalyse qui met en cause de façon élective les garanties identificatoires minimales nécessaires à l'analyste dans son exercice. C'est la possibilité de soutenir une exigence «scientifique» qui procure au clinicien les assises personnelles requises pour soutenir sa fonction clinique. L'auteur utilise Totem et Tabou pour illustrer la démarche épistémologique propre à la psychanalyse, son développement et les progrès théoriques et techniques qu'elle rend possibles.

Par ailleurs, Claudine Vacheret et son équipe, en écho au texte de Jacques Kaës, présenté dans le numéro Printemps 2008 de Filigrane, nous ramènent à un univers académique et présentent l'importance de la formation «au» groupe dans la formation universitaire des psychologues cliniciens de l'Université Lumière-Lyon 2 - en butte, comme d'autres milieux de formation, à la résistance à la psychanalyse —, c'est-à-dire à la transmission de connaissances sur les grands courants théoriques psychanalytiques de groupe. Il s'agit aussi de la formation «par»le groupe, les étudiants participant à des expériences de groupe qui leur sont proposées et, enfin, de l'importance du groupe des pairs dans les séminaires de recherche. Ces trois perspectives donnent au groupe une position centrale dans la formation des futurs praticiens à l'université.

Enfin, Terry Zaloum examine, elle aussi, certains conflits confrontant la pensée psychanalytique à notre époque. Son texte aborde la question des motivations des cliniciens d'expérience à entreprendre une formation psychanalytique. L'auteure évoque son questionnement face à sa pratique et présente les réflexions l'ayant conduite à s'engager dans une formation psychanalytique. Elle souligne les questions sur lesquelles elle juge pertinent de continuer à réfléchir, essentiellement l'importance de l'écoute analytique, le soutien à la capacité contenante du psychothérapeute et le travail sur les résistances. Elle fait ressortir l'utilité de la formation psychanalytique pour développer et soutenir cette disposition à l'écoute tout en maintenant vivante la pensée clinique. Elle partage enfin avec le lecteur sa conviction profonde en la valeur thérapeutique de la psychanalyse, et son désir maintenant assumé de devenir analyste. 
Présentation

\section{Note}

1. A. Green et al, 2005, Argument du colloque «Unité et diversité des pratiques du psychanalyste» tenu à Paris les 14 et 15 janvier 2006 . 